\title{
Multi-Size-Mesh Multi-Time-Step Dispersion-Relation-Preserving Scheme for Multiple-Scales Aeroacoustics Problems
}

\author{
C.K.W. TAM and K.A. KURBATSKII
}

Department of Mathematics, Florida State University, Tallahassee, FL 32306-4510, USA

\begin{abstract}
The optimized high-order Dispersion-Relation-Preserving finite difference scheme is extended so as to be able to compute multiple-scales aeroacoustics problems effectively and efficiently. It is proposed that a multi-size mesh is used in the computation. In implementing the time marching scheme, the computation domain is first partitioned into a number of subdomains. In each subdomain, a single size mesh is used. The mesh size of adjacent subdomain changes by a factor of two. The time step of adjacent subdomains also changes by the same ratio. This choice serves not only to maintain numerical stability but also to avoid unnecessary computations in regions with large size mesh. To pass information between subdomains, special optimized stencils are used at the subdomain interface region. Because rapid changes takes place at the mesh-size-change interfaces, they are likely sources of spurious numerical waves. To prevent the generation and spreading of spurious numerical waves, special artificial selective damping terms are developed for inclusion in the discretized scheme. As an illustration of the efficacy of the multi-size-mesh multi-time-step scheme, it is applied to the simulation of an automobile door cavity tone problem. The computed tone frequencies are found to agree well with experimental measurements.
\end{abstract}

Keywords: Multiple-scales problem, optimized scheme, multi-size-mesh, multi-time-step, cavity tones.

\section{INTRODUCTION}

Most aeroacoustics problems involve multiple length and time scales. In many problems, the intrinsic sizes and scales of the noise sources are an order of magnitude smaller than the acoustic wave length. This large disparity in length scales leads to the classical multiple-scales problem. In jets, the fine scale turbulence or small eddies generate the dominant component of noise that radiates to the sideline directions. The eddies are very small compared to the diameter of the jet. The acoustic wave lengths, on the other hand, are typically more than one jet diameter long. Thus, there is a large difference among these characteristic lengths. Sometimes an aeroacoustics problem becomes a multiple-scales problem because of the change in the physics governing the problem in different parts of the domain. A good example is the shedding of vortices at the edge of a resonator or a solid edge induced by high intensity incident sound waves. Away from the solid surface the fluid is practically inviscid. But viscous effect dominates in the wall region. The oscillatory motion of the incident sound waves induces a very thin viscous layer called the Stokes layer on the solid surface. The Stokes layer rolls up at the edge of a solid surface into vortices that shed periodically. To simulate the vortex shedding process computationally, it is necessary to use very fine mesh on the solid surface and around the edge to resolve the Stokes layer. But away from the solid surface, a coarse mesh with, say, 7 to 8 mesh points per acoustic wave length is all that is needed to capture the sound waves accurately.

One effective way to treat a multiple-scales problem is to use a multi-size mesh. The computation domain is first divided into subdomains. The mesh size is uniform in each subdomain but changes from subdomain to subdomain. Figure 1 shows a typical multi-size mesh. In this case, the mesh size changes by a factor of 2 across the mesh-size-change interface. A factor of 2 change in the mesh size is not an absolute necessity. Experience indicates that it is a good choice, because a larger change may result in a strong artificial discontinuity at the interface. Such a strong discontinuity is undesirable for it tends to become a source of spurious numerical waves and even numerical instability. On the other hand, a smaller change may require several steps of changes to achieve the same total change in mesh size. This will give rise to a large number of mesh-size-change interface, which is also undesirable. 


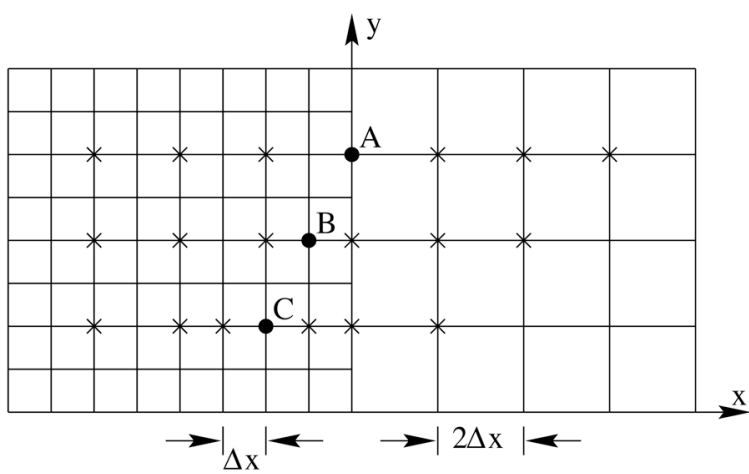

(a)

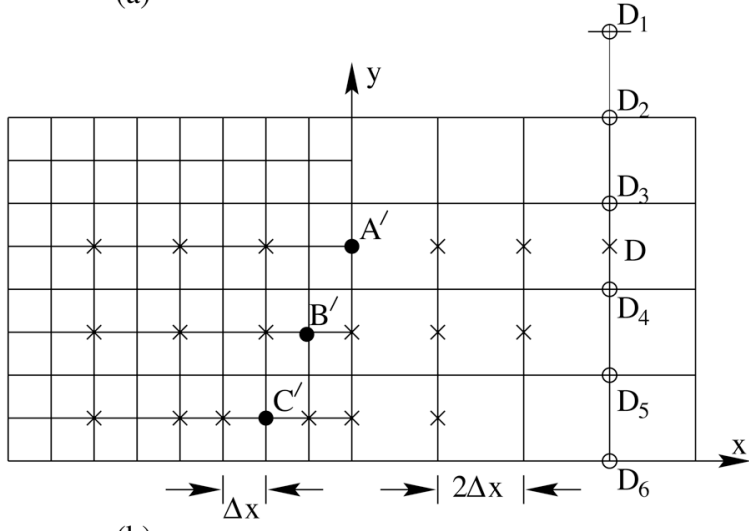

(b)

Figure 1. Special stencils for use in the mesh-size-change buffer region. - mesh points at which finite difference approximation of spatial derivatives is to be found. $\square$ stencil points. $\bigcirc$ points used for interpolation.

When a different size mesh is used, it has a significant impact on the time steps used in a computation. It is well known that numerical instability requirement links $\square t$, the time step, to the mesh size $\square x$, typically by the CFL number. If one uses a single time step marching scheme such as the Runge-Kutta method, then $\square t$ is dictated by the smallest size mesh. This results in an inefficient computation. The optimum situation is to use a $\square t$ that meets the local numerical stability requirement. In such an arrangement, most of the computation is concentrated in regions with the finest mesh, as it should be. In the coarse mesh region, the solution is updated only occasionally with a much larger time step. We will refer to this type of algorithm as "multisize-mesh multi-time-step" schemes. If the mesh-size change is a factor of 2 across mesh block interface, the time step should also change by a factor of 2 to maintain numerical stability. For multi-level time marching schemes, the use of multiple time steps with a factor of 2 change between adjacent mesh block is easy to arrange. This will be discussed in this paper.

In carrying out a multi-size-mesh multi-time-step computation, the computation scheme in each of the uniform mesh subdomain is the same as if a single size mesh is used throughout the entire computation domain. The exception is for a narrow buffer region at the subdomain interface. In the buffer region, special stencils are to be used so that information on the solution can be transmitted through accurately. Also, as stated before, any surface of discontinuity, no matter if it is a mesh-size-change interface or an internal or external boundary, is a likely source of short spurious numerical waves. To suppress the generation of these unwanted waves, special artificial selective damping stencils must be incorporated into the computation scheme for mesh points in the interface region.

Because of the need to propagate waves over long distances, computational aeroacoustics (CAA) schemes must ideally be nondispersive and nondissipative. To meet these requirements, the use of high-order schemes becomes a necessity. In the literature, there are a number of high-quality high order schemes (Hirsh, 1975; Lele, 1992; Casper, 1994; Gaitonde et al., 1997; Gaitonde and Visbal, 1999). Recently Tam and Webb (1993) developed a high-order finite difference method known as the Dispersion-Relation-Preserving (DRP) scheme specifically designed for CAA and wave propagation applications. The scheme is a central difference scheme and thus has no intrinsic numerical damping. Numerical dispersion is controlled by the group velocity of the numerical method. The stencil coefficients of the DRP scheme are chosen not only to ensure that the computation has a wide resolved bandwidth in wavenumber space but also the group velocity is equal to wave propagating velocity of the physical system. One unique characteristic of the scheme is that it would automatically preserve the dispersion relations of the partial differential equations in the resolved range of wavenumber and frequency. This assures that the wave mode supported by the original equations are reproduced faithfully by the finite difference equations. In this paper the DRP scheme will be used in each of the subdomains. Special stencils needed at the buffer region of the mesh-size-change interface will be designed by an optimization procedure similar to the approach of Tam and Webb. These stencils will also possess the dispersion relation preserving characteristic. Thus the resulting algorithm is a truely multi-size-mesh multi-time-step DRP scheme.

The rest of this paper is as follows. In Section 2, the design of special spatial stencils for the buffer region will be discussed. Optimized stencil coefficients are determined. The wave propagation characteristics of the stencils as well as their accuracies in wavenumber space are examined. In Section 3, a multi-time-step marching scheme is 
formulated. The use of multi-time-step method is capable of reducing the computation time of some problems by a large factor. Details of how multitime-step is to be implemented are described.

Artificial selective damping stencils for the buffer region are considered in Section 4. Optimized damping stencil coefficients are provided. Finally, the multi-size-mesh multi-time-step DRP scheme is applied to the study of tone generation induced by flow over an automobile door cavity. This example serves to illustrate a practical application of the computational method.

\section{SPATIAL STENCILS FOR USE IN MESH- SIZE-CHANGE BUFFER REGION}

If a factor of two increase in the mesh size between adjacent blocks is used, then every other mesh line in the fine mesh block continues into the coarse mesh block as shown in figure 1 . The remaining set of mesh lines terminates at the mesh-size-change interface (see figure 1). To compute the $x$-derivative for points on the coarse grid, including points on the interface such as point $A$, the 7-point central difference DRP scheme may be used even though part of the stencil is extended into the fine mesh region. For mesh points on the fine grid, again the 7point central difference DRP scheme may be used except for the first three columns (or rows) of mesh points right next to the coarse grid. For points on the continuing mesh lines such as points $B$ and $C$ in figure 1 , special central difference stencils as indicated are to be used. These stencils may be written in the form,

$$
\begin{aligned}
& \left.\square \frac{\partial f}{\partial x}\right|_{B} \square \frac{1}{\square x} \prod_{j=\square 3}^{3} a_{j}^{B} f_{j}^{B} \\
& \left.\square \frac{\partial f}{\partial x}\right|_{C} \square \frac{1}{\square x} \square_{j=\square 3}^{3} a_{j}^{C} f_{j}^{C}
\end{aligned}
$$

To find the stencil coefficients $a_{j}^{B}$ and $a_{j}^{C} \quad(j=-3$ to 3 ), the optimization procedure of Tam and Webb (1993) will be followed. Now first consider the stencil for point $C$. It will be assumed that $f(x)$ has a Fourier transform $\tilde{f}(\square)$ with absolute value $A(\square)$ and argument $\square(\square)$; i.e.,

$$
A(\square)=|\tilde{f}(\square)|, \quad \square(\square)=\arg [\tilde{f}(\square)] .
$$

The Fourier transform may be written as,

$$
f(x)=\bigsqcup_{\square}(\square) e^{i[\square x+\square(\square)]} d \square .
$$

In other words, $f(x)$ is made up of a superposition of simple waves, $f_{a}(x)$, of the form,

$$
f_{\square}(x)=e^{i[\square x+\square(\square)]}
$$

weighted by $A(\square)$. We may now examine the approximation (2) on each simple wave component of $f(x)$. Upon substituting $f_{a}(x)$ into (2), the finite difference approximation becomes,

$$
\square f_{\square}(x) \square \square f_{\square}(x)
$$

where

$$
\begin{aligned}
\square= & \frac{2}{\square x}\left[a_{1}^{C} \sin (\square \square x)+a_{2}^{C} \sin (2 \square \square x)\right. \\
& \left.+a_{3}^{C} \sin (4 \square \square x)\right]
\end{aligned}
$$

is the wavenumber of the finite difference stencil. In deriving (5), the antisymmetric condition, $a_{\square j}^{C}=\square a_{j}^{C}$, has been invoked.

We will impose the condition that (2) or (5) be of order $(\square x)^{4}$ accurate. By means of Taylor series expansion, this condition yields the following restrictions on the stencil coefficients,

$$
\begin{aligned}
& 2\left(a_{1}^{C}+2 a_{2}^{C}+4 a_{3}^{C}\right)=1 \\
& a_{1}^{C}+2^{3} a_{2}^{C}+4^{3} a_{3}^{C}=0
\end{aligned} .
$$

The coefficients will now be chosen so that the error of using $\Pi \Pi x$ to approximate $\square \square x$ over the band of wavenumber $|\square \square x|<\square, E$, is minimum subjected to the conditions (6). For this purpose, the error is defined as

$$
\left.E=\prod_{0}^{\square} \square \square x \square \square \square x\right]^{2} d(\square x) .
$$

The condition for a minimum is given by (after eliminating $a_{2}^{C}$ and $a_{3}^{C}$ by (6)

$$
\frac{d E}{d a_{1}^{C}}=0 .
$$

An extensive numerical study of the effects of the choice of $\square$ on $\square(\square)$ and $d \Pi / d \Pi$ has been carried out. Based on the numerical results, it is deemed that a good choice of the values of $\square$ is 1.0. For this choice of $\square$ the stencil coefficients are,

$$
\begin{aligned}
& a_{0}^{C}=0.0 \\
& a_{1}^{C}=\square a_{\square 1}^{C}=0.726325187522 \\
& a_{2}^{C}=\square a_{\square 2}^{C}=\square 0.120619908868 \\
& a_{3}^{C}=\square a_{\square 3}^{C}=0.003728657553
\end{aligned} .
$$

Figures 2 and 3 show the corresponding $\square(\square)$ and $d \Pi / d \Pi$ curves. The resolution and dispersion 
characteristics of the stencil lie in-between those of the central difference DRP scheme on the two sides of the interface.

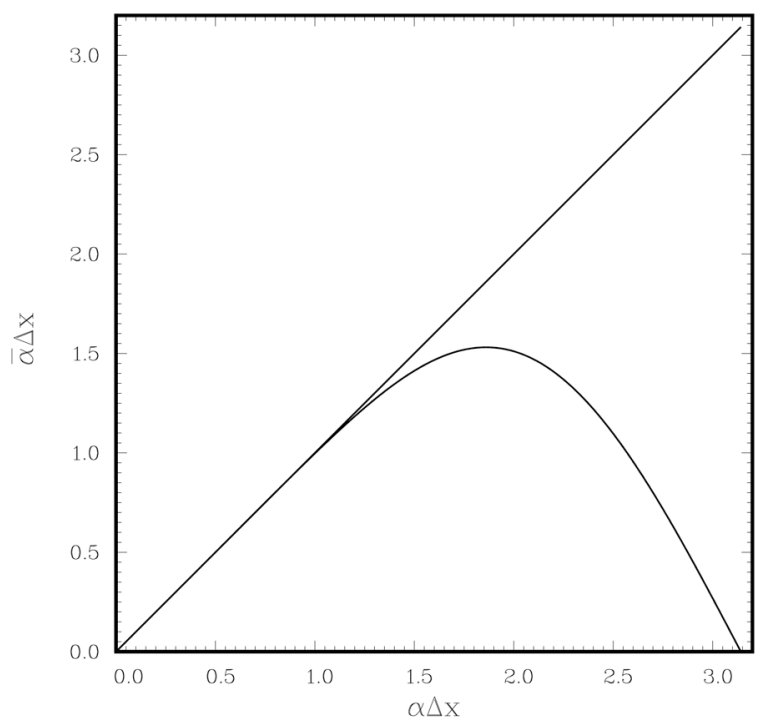

Figure 2. Dependence of $\Pi \sqcap x$ on $\square \square x$ for stencil $C$. $\square=1.0$.
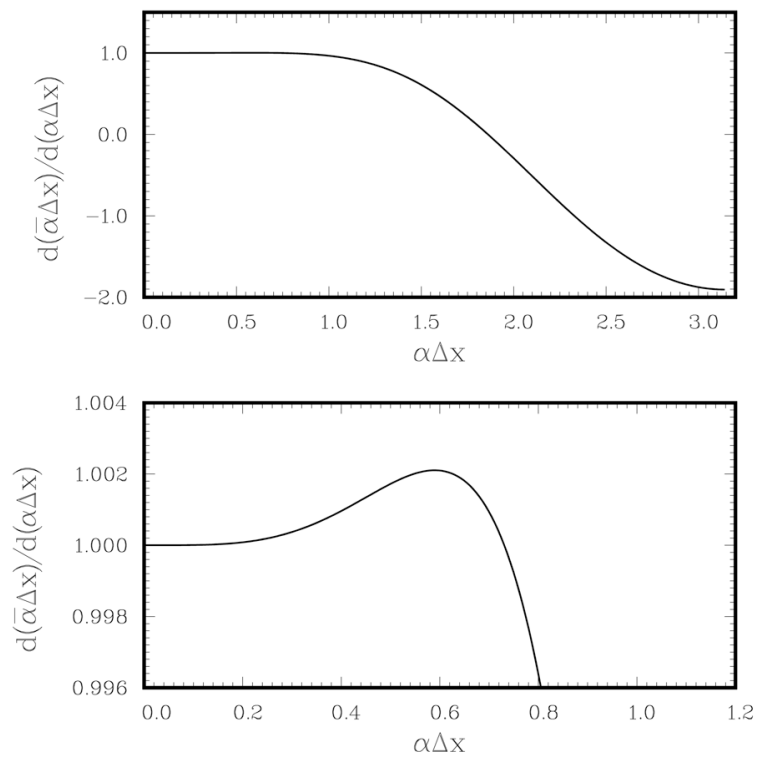

Figure 3. The $d(\Pi \sqcap x) / d(\square \square x)$ curve for stencil $C$. $\square=1.0$.

On proceeding as for stencil $C$, the stencil coefficients for stencil $B$ may be found. For this case, it is recommended that the value $\square=0.85$ be used. The stencil coefficients are,

$$
\begin{aligned}
& a_{0}^{B}=0.0 \\
& a_{1}^{B}=\square a_{\square 1}^{B}=0.595328177715 \\
& a_{2}^{B}=\square a_{\square 2}^{B}=\square 0.037247422191 \\
& a_{3}^{B}=\square a_{\square 3}^{B}=0.003282817772
\end{aligned} .
$$

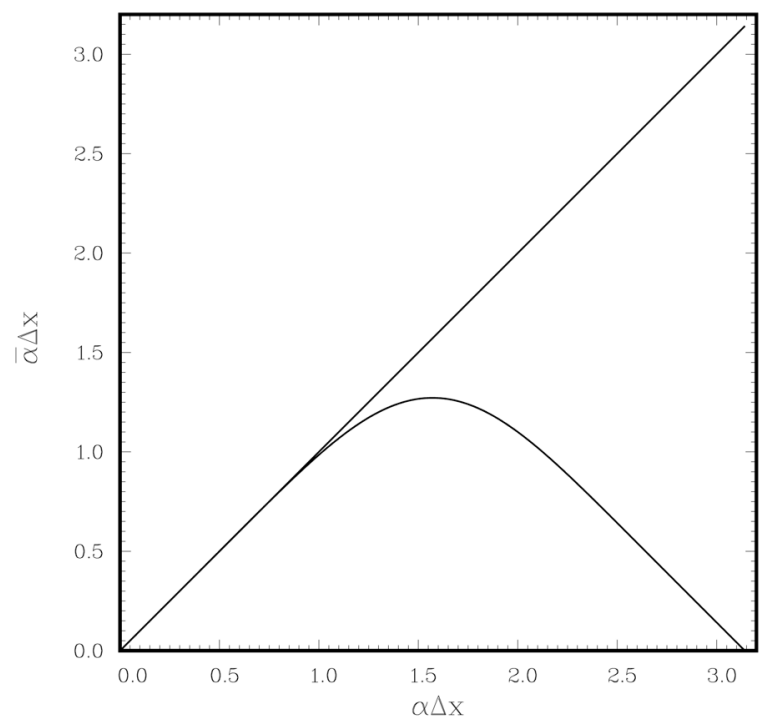

Figure 4. Dependence of $\Pi \sqcap x$ on $\square \square x$ for stencil $B . \square=0.85$.
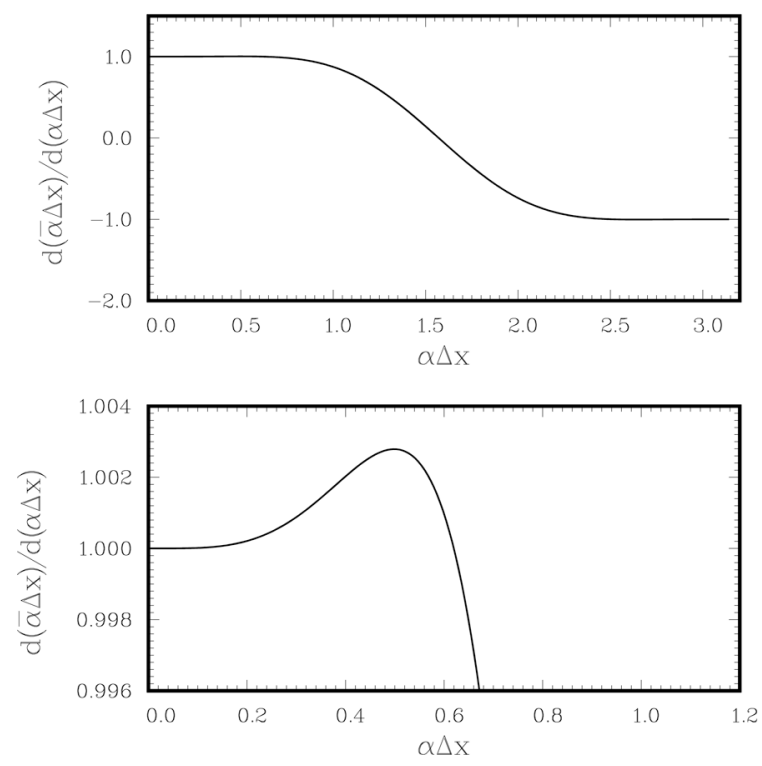

Figure 5. The $d(\Pi \sqcap x) / d(\square \square x)$ curve for stencil $B . \square=0.85$.

Figures 4 and 5 show the corresponding $\square(\square)$ and $d \square / d \square$ curves for this stencil. Again the resolution and dispersion characteristics lie in-between those of the central difference DRP scheme on the two sides of the interface.

For mesh points lying on the terminating mesh lines such as points $A^{\prime}, B^{\prime}$ and $\mathrm{C}^{\prime}$ of figure 1 , the same stencils as for $A, B$ and $C$ may be used, However, the stencils extend into points in the coarse mesh region where the solution is not computed. To obtain the values of the solution at these points, such as point $D$, it is recommended that interpolation be used. The interpolation stencil, a symmetric optimized stencil is preferred (see Tam and Kurbatskii, 2000a), makes use of the values of the adjacent six mesh points, $D_{1}$ to $D_{6}$, as shown in figure 
1. In actual computation, the interpolation step is executed only after the solution on the coarse mesh region has been advanced by a time step. The interpolated values allow the solution on the fine mesh side to continue advancing in time.

\section{TIME-MARCHING STENCIL FOR USE IN MESH-SIZE-CHANGE BUFFER REGION}

The time marching step, $\square t$, is constrained by the numerical stability or accuracy requirement of a computation scheme. In general, the stability or accuracy requirement links $\square t$ directly to the mesh size $\square x$. Thus in a region with large mesh size, a large $\square t$ may be used. When more than one mesh size is used. The optimum strategy is to use the largest time step permissible in each region. It follows, if the mesh size changes by a factor of two in adjacent domains, the time step should also change by the same ratio. Figure 6 shows the time levels of the computation in the mesh-size-change buffer region. In the fine mesh region on the left, the time step is $\square t$. In the coarse mesh region on the right, the time step is $2 \square t$. Effectively, the fine mesh region is computed twice as often as the coarse mesh region.

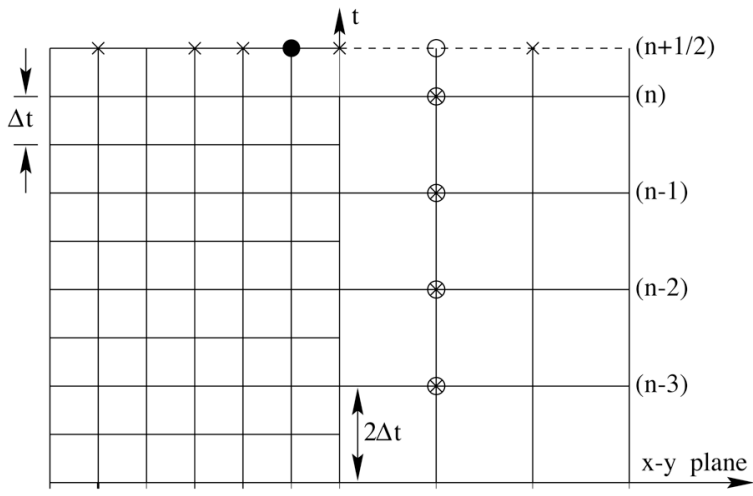

Figure 6. Special time-marching stencil for use in the mesh-sizechange buffer region. $\bigcirc$ mesh point at which solution is to be found at half-time level. stencil points.

Suppose the solution is known at time level $n$ (see figure 6), the solution in the fine mesh region may be advanced by a time step $\square t$ to time level $(n+(1 / 2))$ in the usual way. Once this is completed, the next step is to advance the solution to time level $(n+1)$ in both the fine and coarse mesh regions. It is straightforward to carry out this step in the coarse mesh region by using the solution on time levels $n,(n-1),(n-2)$ and $(n-3)$. However, for points in the buffer region on the fine mesh side, the stencils extend into the coarse mesh region. However, there is no information at the $(n+(1 / 2))$ time level at these points on the coarse mesh side. To provide the needed information of the solution, it is necessary to compute the solution at the time level $(n+(1 / 2))$ for the first two rows or columns of the mesh point on the coarse mesh side based on the solution at time levels $n,(n-1),(n-2)$ and $(n-3)$. To advance the solution by a half time step as shown in figure 6 , the following four level scheme may be used.

$$
\mathbf{u}_{\ell, m}^{\left(n+\frac{1}{2}\right)}=\mathbf{u}_{\ell, m}^{(n)}+(2 \square t) \prod_{j=0}^{3} b_{j}^{*} \mathbf{K}_{\ell, m}^{(n \square j)}
$$

where $\mathbf{K}=d \mathbf{u} / \mathrm{dt}$ is given by the governing equation. $(\ell, m)$ are the spatial indices.

To find the stencil coefficients $b_{j}^{*}$ of (9), one may

consider $\mathbf{u}$ to be made up of a Fourier spectrum of simple harmonic components of the form $e^{-i \square t}$ where $\square$ is the angular frequency. The effect of time marching algorithm (9) on each Fourier component of frequency $\square$ can be analyzed by substituting $\mathbf{u}_{(\ell, m)}^{(n)}=\mathbf{u}_{(\ell, m)}(\mathbf{t})=\mathbf{A} e^{\square i \square n(2 \square t)}$ into (9). It is easy to find,

$$
\frac{d \mathbf{u}_{\ell, m}}{d t}=\frac{\left(e^{\square i \square \square t} \square 1\right)}{(2 \square t) \bigsqcup_{j=0}^{3} b_{j}^{*} e^{2 i j \square \square t}} \mathbf{u}_{\ell, m}
$$

Since $\mathrm{d} \mathbf{u}_{\ell, m} / \mathrm{dt}=-\mathrm{i} \square \mathbf{u}_{\ell, m}$, the factor, on the right side of (10) multiplying $\mathbf{u}_{\ell, m}$, must be equal to $\Pi i \Pi$ to where $\Pi$ is the angular frequency of the time marching finite difference scheme. Thus one finds,

$$
\square \square t=\frac{i\left(e^{\square i \square \square t} \square 1\right)}{2 \prod_{j=0}^{3} b_{j}^{*} e^{2 i j \square \square t}} .
$$

There are four coefficients, namely, $b_{0}^{*}, b_{1}^{*}, b_{2}^{*}$ and $b_{3}^{*}$. Here the condition that $\square \square \square$ be accurate to order $(\square t)^{2}$ is imposed. By expanding (11) by Taylor series, it is straightforward to find that the four coefficients are related by the following three conditions.

$$
\begin{gathered}
\bigsqcup_{j=0}^{3} b_{j}^{*}=\frac{1}{2} \\
\bigsqcup_{j=0}^{3} j b_{j}^{*}=\square \frac{1}{8} \\
\prod_{j=0}^{3} j^{2} b_{j}^{*}=\frac{1}{24} .
\end{gathered}
$$

There is no loss of generality in assuming that $b_{0}$ is the remaining free parameter. The value of $b_{0}$ is 
chosen so that $\Pi$ is a good approximation of $\square$ over the frequency band $-\square \leq \square \square t \leq \square$ This is done by minimizing the integral

$$
\begin{aligned}
E\left(b_{0}\right)= & \underset{\square \square}{\square}\left\{\square[\operatorname{Re}(\square \square t) \square \square \square t]^{2}\right. \\
& \left.+(1 \square \square)[\operatorname{Im}(\square \square t)]^{2}\right\} d(\square \square t)
\end{aligned}
$$

where $\square$ is the weight parameter. The condition for minimization is $d E / d b_{0}=0$.

A numerical study of the real and imaginary parts of $(\square \square t \square \square \square t$ ) for different choice of $\square$ and $\square$ has been carried out. Based on the results of this study, the values $\square=0.5$ and $\square=0.42$ are chosen for use. For this choice of parameters, the optimized coefficients are,
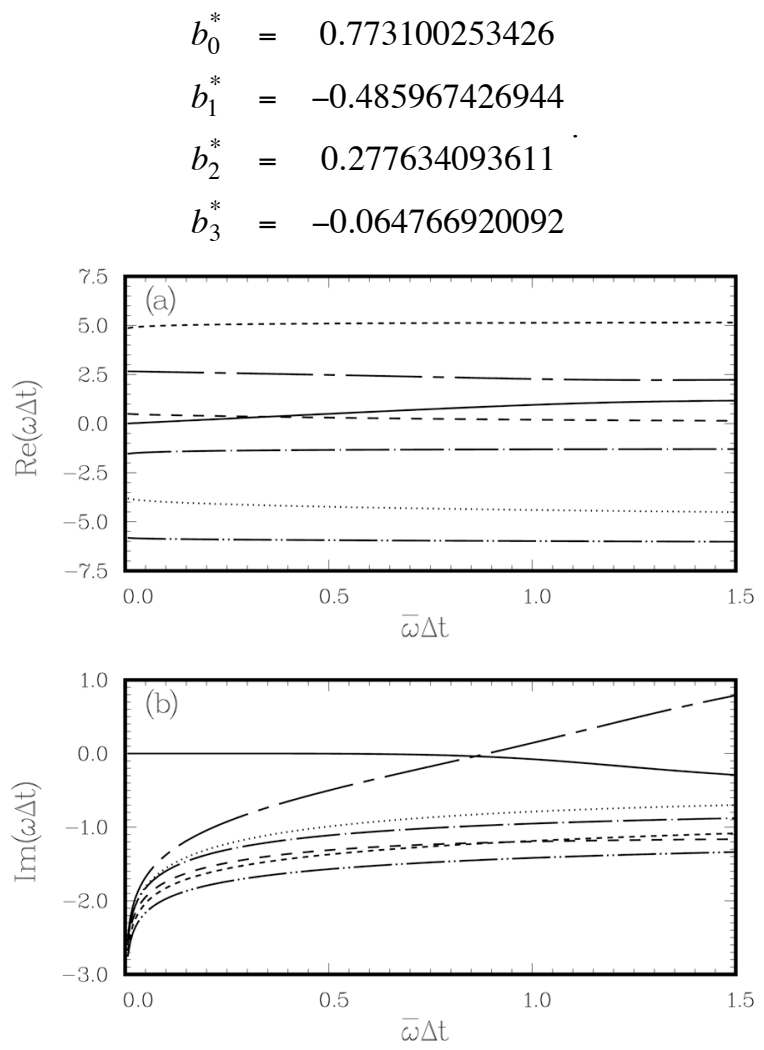

Figure 7. The dependence of the seven roots of $\square \square t$ on $\Pi \prod t$. (a) Real part. (b) Imaginary part.

For a given $\Pi \Pi t,(11)$ yields 7 roots of $\square \square t$. As in the original DRP scheme, only one of the roots yields the physical solution. All the other roots are spurious and should be damped if the scheme is to be stable. Figure 7 shows the dependence of the roots on $\square \square t$. The scheme is stable if $\square \square t<0.88$. If $\square \square t$ is restricted to less than or equal to 0.19 , then the damping rate, $\operatorname{Im}(\square \square t)$, of the physical root is less than $0.78 \square 10^{-5}$, which is quite small. The accuracy of this scheme when restricted to this range is comparable to that of the standard DRP scheme.

\section{ARTIFICIAL SELECTIVE DAMPING}

In numerical computation, surfaces of discontinuity, such as at a mesh-size-change interface, are potential sources of spurious numerical waves. For this reason, it is necessary to add artificial selective damping in the buffer region of the interface. For points $A$ and $A^{\prime}$ the 7-point damping stencils discussed in Tam, Webb and Dong (1993) and Tam (1995) may be used. For points $B$ or $B^{\prime}, C$ or $C^{\prime}$ special damping stencils are necessary.

Consider the $x$-momentum equation of the linearized Euler equations.

$$
\frac{\partial u}{\partial t}+\square=0
$$

The discretized form of (16) at point $B$ including the artificial selective damping terms is

$$
\left.\frac{\square d u}{{ }^{d} d t}\right]_{B}+\square=\square \frac{\square_{a}}{(\square x)^{2}} \square_{j=\square 3}^{3} d_{j}^{B} u_{B, j}
$$

where the damping stencil coefficients satisfy the symmetric condition $d_{\square j}^{B}=d_{j}^{B} . \square_{a}$ is the artificial kinematic viscosity. $\square x$ is the mesh size at $B$.

Let $u$ be a single Fourier component $\hat{u}(t) e^{i \square \ell \square x}$. Substitution into (17) yields

$$
\frac{\square}{d t} \mathrm{O}_{B}+\square=\square \frac{\square_{a}}{(\square x)^{2}} D^{B}(\square \square x) u .
$$

Upon integration, it is found

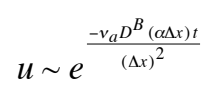

where the damping function $D^{B}(\square \square x)$ is given by,

$$
\begin{aligned}
D^{B}(\square \square x)= & d_{0}^{B}+2\left[d_{1}^{B} \cos (\square \square x)\right. \\
& +d_{2}^{B} \cos (3 \square \square x) \\
& \left.+d_{3}^{B} \cos (5 \square \square x)\right]
\end{aligned}
$$

As in Tam, Webb and Dong (1993), we will require that there is no damping if $u$ is a constant (or $\square \square \mathrm{x} \square 0$ ). This leads to

$$
d_{0}^{B}+2 \square_{j=1}^{3} d_{j}^{B}=0 .
$$

The normalization condition is $D^{B}(\square)=1.0$ or

$$
d_{0}^{B} \square 2\left(d_{1}^{B}+d_{2}^{B}+d_{3}^{B}\right)=1 .
$$


In addition, it is intended to select the remaining parameters such that (20) is a good approximation to a Gaussian function of half width $\square$ centered at $\square \square x=\square$. Specifically, the integral

$$
\left.\prod_{0}^{\square} D^{B}(\square \square x) \square e^{\square(\ln 2)(\square \square x \square \square / \square)^{2}}\right]^{2} d(\square \square x)
$$

is to be minimized. Numerical experiments suggest that a good choice of $\square$ and $\square$ are:

$$
\square=0.8, \quad \square=0.2 \square .
$$

This yields the following damping stencil coefficients,

$$
\begin{aligned}
& d_{0}^{B}=0.5 \\
& d_{1}^{B}=d_{\square 1}^{B}=\square 0.294977493296 \\
& d_{2}^{B}=d_{\square 2}^{B}=0.052389707989 \\
& d_{3}^{B}=d_{\square 3}^{B}=\square 0.007412214693
\end{aligned} .
$$

The damping function corresponding to this stencil is shown in figure 8.

On following the above steps, the coefficients of the damping stencil for points $C$ or $C^{\prime}$ may be found. In this case, numerical experiments suggest the choice of $\square=0.7$ and $\square=0.2 \square$. The damping stencil coefficients are,

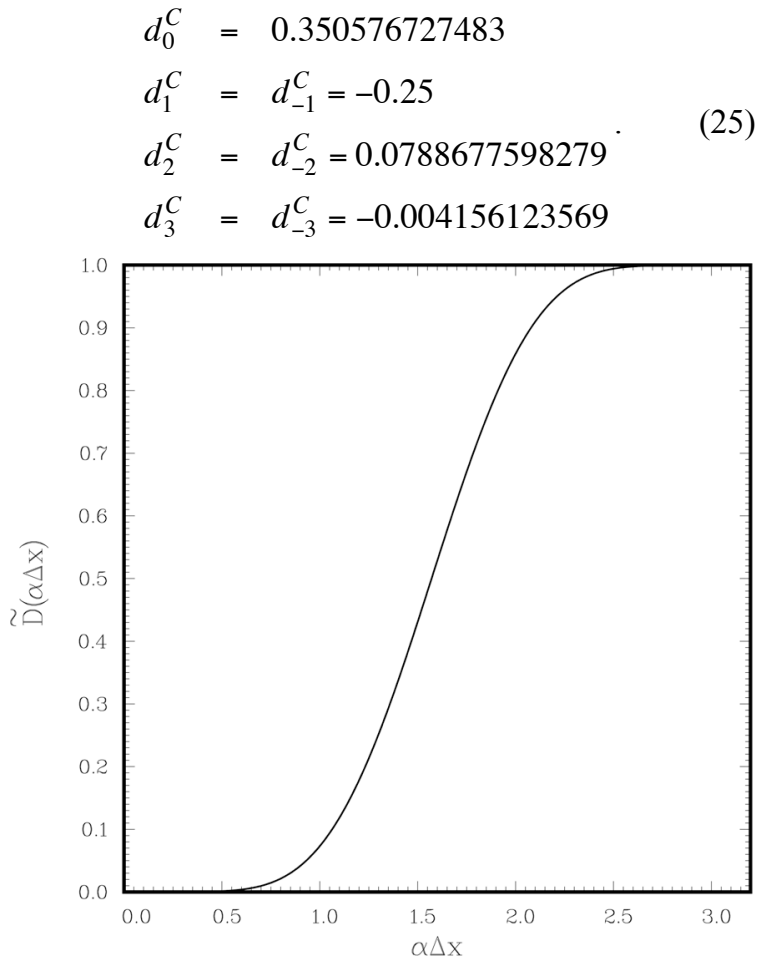

Figure 8. Damping function for stencil $B$. $\square=0.8, \square=0.2 \square$.

The damping function is shown in figure 9 .

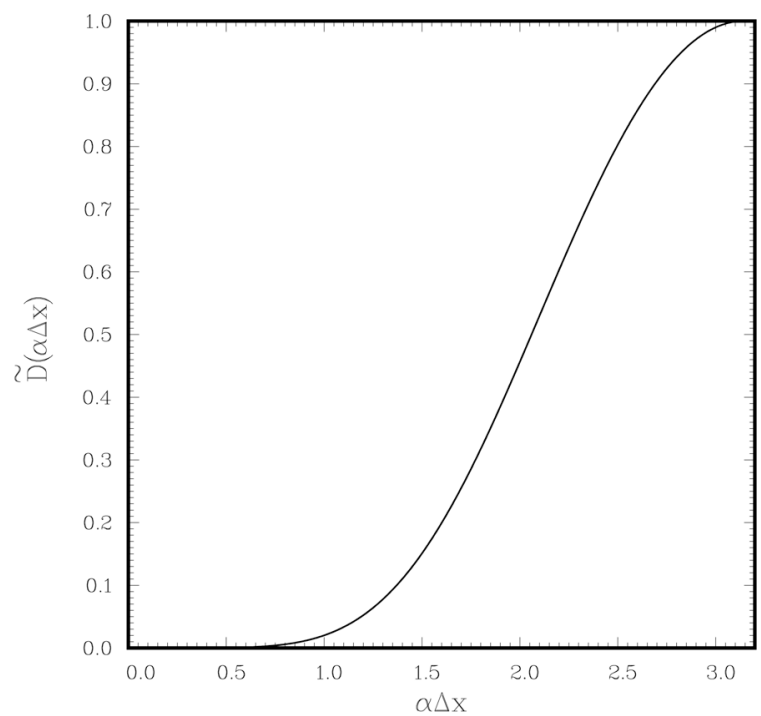

Figure 9. Damping function for stencil C. $\square=0.7, \square=0.2 \square$.

Spurious numerical waves are often generated at the mesh-size-change interface. For boundary layer or similar problems, the mesh size is reduced toward the wall. This mesh arrangement could lead to the amplification of short spurious waves as described below. The continuous reduction of mesh size toward the wall makes it possible for grid-to-grid oscillations to be trapped between one of the nested mesh layers and the wall. This provides an environment for the grid-to-grid oscillations to grow. If left unchecked, it could lead to numerical instability. For a nested mesh layer of this category, artificial selective damping must be included across the entire layer. For an estimate of the magnitude of the mesh Reynolds number that should be imposed in the computation, it is noted that for grid-to-grid oscillations $(\square \square x=\square)$, the time, $T$, taken for the short waves to propagate from one side of the layer to the other side, is equal to the distance travelled divided by the speed of propagation. Here the group velocity of the spurious grid-to-grid oscillations is taken to be approximately equal to twice the speed of sound $a_{0}$.

$$
T=\frac{N \square x}{2 a_{0}}
$$

where $N$ is the number of mesh points across the width of the layer, $\square x$ is the mesh size. The total damping in this time period is given by (19) with $\tilde{D}(\square)=1.0$; i.e.,

$$
e^{\square \frac{\square a T}{(\square x)^{2}}}=e^{\square \frac{N}{2 R \square}}
$$

where $R_{\square}=\square x a_{0} / \square$ is the mesh Reynolds number. It is desirable to have this factor equal to $10^{-2}$ or less. On setting the factor of (27) to $10^{-2}$, a criterion for the 
choice of the mesh Reynolds number is established, namely,

$$
\frac{N}{R_{\square}} \geq 9.2
$$

As an example, if the layer has 20 mesh points, $N=20$, then $R_{\square}^{\square 1} \geq 0.46$, say 0.5 , should be used. This value is much larger than the value recommended for general background damping, which is $R_{\square}^{\square 1} \geq 0.05$. (Note: away from the solid boundaries a smaller and smaller inverse mesh Reynolds number should be used.) Formula (28) also suggests that any uniform subdomain in a multi-size-mesh computation should not have much fewer than 20 mesh points. This is to avoid the necessity of using excessively large artificial damping.

\section{APPLICATION}

To illustrate the effectiveness of multi-size-mesh multi-time-step computing, the automobile door cavity tone problem of the Third CAA Workshop on Benchmark Problems is considered (Dahl, 2000).

The benchmark problem specifies a turbulent boundary layer flow over the cavity. To properly model and compute the turbulent boundary layer flow and its interaction with the cavity is a task that will require extensive time and effort. Here a laminar boundary layer is considered instead. It is believed that the cavity tone frequency would most likely be about the same whether the flow is turbulent or laminar, but the tone intensity is expected to be different.

A boundary layer flow will definitely be laminar if $R_{\square^{*}}<600$. This is the Reynolds number below the stability limit of the Tollmien-Schlichting waves. In modern facilities with low free stream turbulence and sound, a boundary layer may remain laminar if $R_{\square^{*}}$ is larger than 600 but less than 3400 . For a free stream velocity of $50.9 \mathrm{~m} / \mathrm{s}$ and $26.8 \mathrm{~m} / \mathrm{s}$ (velocities prescribed by the benchmark problem), this corresponds to a boundary layer thickness of $2.9 \mathrm{~mm}$ and $5.5 \mathrm{~mm}$, respectively. In this investigation, we will, therefore, restrict our consideration to a boundary layer thickness of $2 \mathrm{~mm}$ and $1 \mathrm{~mm}$ for flow velocities of $50.9 \mathrm{~m} / \mathrm{s}$ and $26.8 \mathrm{~m} / \mathrm{s}$.

\subsection{The Computation Domain and Grid Design}

The computation domain is shown in figure 10. It is designed primarily for the case $U=50.9 \mathrm{~m} / \mathrm{s}$ and a boundary layer thickness $\square=2 \mathrm{~mm}$. In the actual computation, the outside dimensions of the computation domain change somewhat with flow velocity and boundary layer thickness.

In the cavity opening region, viscous effects are important. To capture these effects, a fine mesh is needed. Away from the cavity, the disturbances are mainly acoustic waves. By using the DispersionRelation-Preserving (DRP) scheme in the computation, only a very coarse mesh would be necessary in the acoustic region. The mesh design is dictated by these considerations. The computation domain is divided into a number of subdomains as shown in figure 10 . The finest mesh with $\square x=\square y=0.0825 \mathrm{~mm}$ is used in the cavity opening region. The mesh size increases by a factor of 2 every time one crosses into the next subdomain. The mesh size in the outermost subdomain is 32 times larger than the finest mesh.

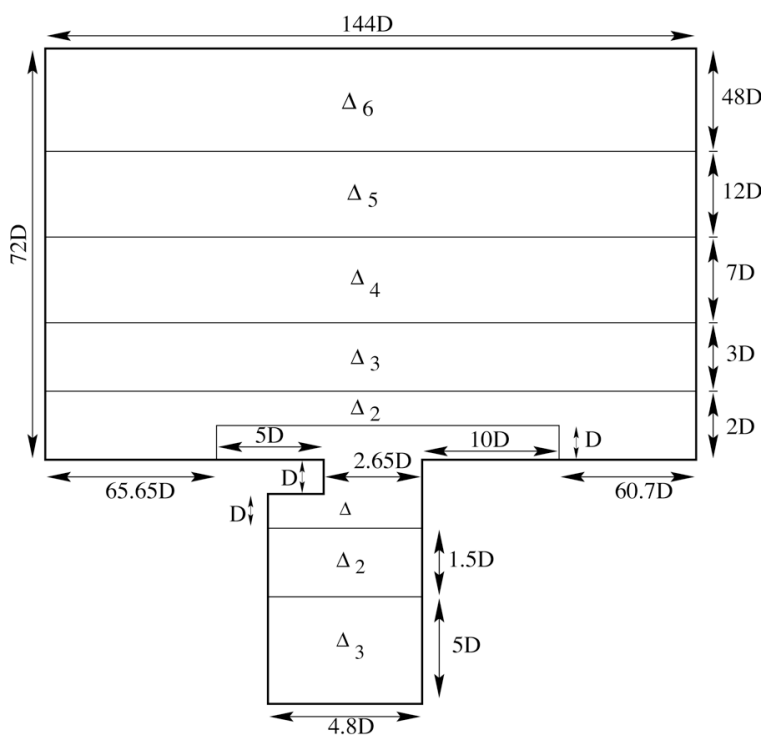

Figure 10. Computation domain showing the division into subdomains and their mesh size. $D=3.3 \mathrm{~mm}$.

\subsection{The Governing Equations and the Computational Algorithm}

The governing equations are the compressible Navier-Stokes equations in two-dimensions.

$$
\begin{gathered}
\frac{\partial \square}{\partial t}+\square \frac{\partial u_{j}}{\partial x_{j}}+u_{j} \frac{\partial \square}{\partial x_{j}}=0 \\
\frac{\partial u_{i}}{\partial t}+u_{j} \frac{\partial u_{i}}{\partial x_{j}}=\square \frac{1}{\square} \frac{\partial p}{\partial x_{i}}+\frac{1}{\square} \frac{\partial \square_{i j}}{\partial x_{j}} \\
\frac{\partial p}{\partial t}+u_{j} \frac{\partial p}{\partial x_{j}}+\square \frac{\partial u_{j}}{\partial x_{j}}=0 \\
\square_{i j}=\square \frac{\square_{0} \partial u_{i}}{\partial x_{j}}+\frac{\partial u_{j}}{\partial x_{i}} \text { 目 }
\end{gathered}
$$


In this study, the above equations are solved in time by the multi-size-mesh multi-time-step DRP algorithm. In each subdomain of figure 10, the equations are discretized by the DRP scheme of Tam and Webb (1993). At the mesh size change boundaries, special stencils as given in Section 2 are used. The time-steps of adjacent subdomains differ by a factor of 2 just as the mesh size. By using the multi-size-mesh multi-time-step algorithm most of the computation effort and time are spent in the opening region of the cavity where the resolution of the unsteady viscous layers is of paramount importance.

\subsection{Artificial Selective Damping}

Artificial selective damping is added to the time marching DRP scheme to eliminate spurious short waves and to prevent the occurrence of numerical instability. The damping stencil with a damping curve of half-width $0.2 \square$ is used for background damping. Near the solid walls or the outer boundaries where a 7-point stencil does not fit, a 5- or 3-point stencil as provided in by Tam (1995) is used instead. For general background damping an inverse mesh Reynolds number $\left(R_{\square}^{\square 1}=\square_{a} /(a \square x)\right.$ where $\square_{a}$ is the artificial kinematic viscosity and $a_{\infty}$ is the speed of sound) of 0.05 is used everywhere. Along walls and mesh change interfaces, additional damping is included. The added damping has an inverse mesh Reynolds number distribution in the form of a Gaussian function with the maximum value at the wall or interface and a half-width of four mesh points. On the wall, the maximum value of $R_{\square}^{\square 1}$ is set equal to 0.15 . The corresponding value at a meshsize-change interface is 0.3 . There are three external corners at the cavity opening. They are likely sites at which short spurious waves are generated. To prevent numerical instability from developing at these points, additional artificial selective damping is imposed. Again a half-width of 4 mesh point Gaussian distribution of the inverse mesh Reynolds number centered at each of these points is used. The maximum value of $R_{\square}^{\square 1}$ at these points is set equal to 0.35. By implementing artificial selective damping distribution as described, it is our experience that no numerical instability nor excessive short spurious waves have been found in all the computations.

\subsection{Numerical Boundary Conditions}

Along the solid surfaces of the cavity and the outside wall, the no-slip boundary condition is enforced by the ghost point method of Tam and Dong (1994).
Along the external boundary region ( 3 mesh points adjacent to the boundary), the flow variables are split into a mean flow and a time dependent component. The mean flow, with a given boundary layer thickness, is provided by the Blasius solution. The time dependent part of the solution is the only portion of the solution that is computed by the time marching scheme (for the split variable method, see Tam (1998)). The boundary conditions used for the computation are as follows. Along the top and left external boundaries the asymptotic radiation boundary conditions of Tam and Webb (1993) are imposed. Along the right boundary, the outflow boundary conditions (Tam and Webb, 1993) are used.

\subsection{Numerical Results}

In this work, the time-marching computation uses the time independent boundary layer solution without the cavity as the initial condition. Computation continues until a time periodic state is reached.

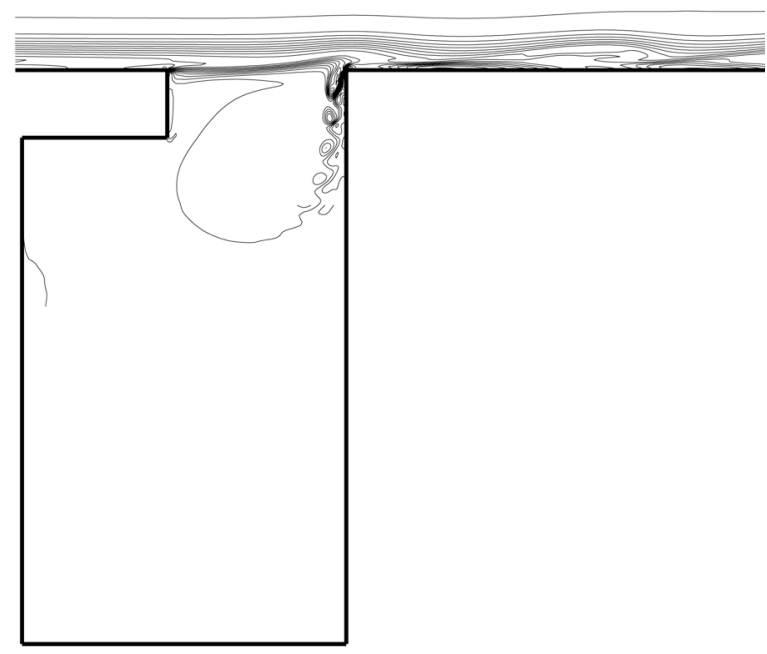

Figure 11. Instantaneous vorticity contours showing the shedding of small vortices at the trailing edge of the cavity. $U=50.9 \mathrm{~m} / \mathrm{s}$, I $=2 \mathrm{~mm}$.

The characteristic features of the flow in the vicinity of the cavity opening and the acoustic field can be found by examining the instantaneous vorticity, steamlines and pressure contours. Figure 11 shows a plot of the instantaneous vorticity contours for the case $U=50.9 \mathrm{~m} / \mathrm{s}$ and $\nabla=2.0 \mathrm{~mm}$. As can easily be seen, vortices are shed periodically at the trailing edge of the cavity. Some shed vortices move inside the cavity. Other vortices are shed into the flow outside. The shed vortices outside are convected downstream by the boundary layer flow. These convected vortices are clearly shown in the pressure contour plot of figure 12. They form the low pressure centers. These vortices persist over a rather long distance and eventually dissipated by viscosity. 
Figure 13 shows the instantaneous streamline pattern. It is seen that the flow at the mouth of the cavity is completely dominated by that of a single large vortex. Below the large vortex, another vortex of opposite rotation often exists. The position of this vortex changes from time to time and does not always attach to the cavity wall. The far field pressure contour pattern is shown in figure 14. This pattern is the same as that of a monopole acoustic source in a low subsonic stream. That the noise source is a monopole and not a dipole is consistent with the model of Tam and Block (1978). The sound is generated by flow impinging periodically at the trailing edge of the cavity.

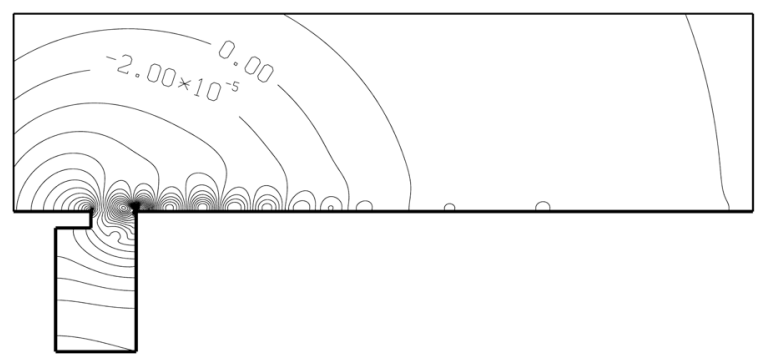

Figure 12. Near field pressure contours showing the convection of shed vortices along the outside wall. $U=50.9 \mathrm{~m} / \mathrm{s}, \square=2 \mathrm{~mm}$.

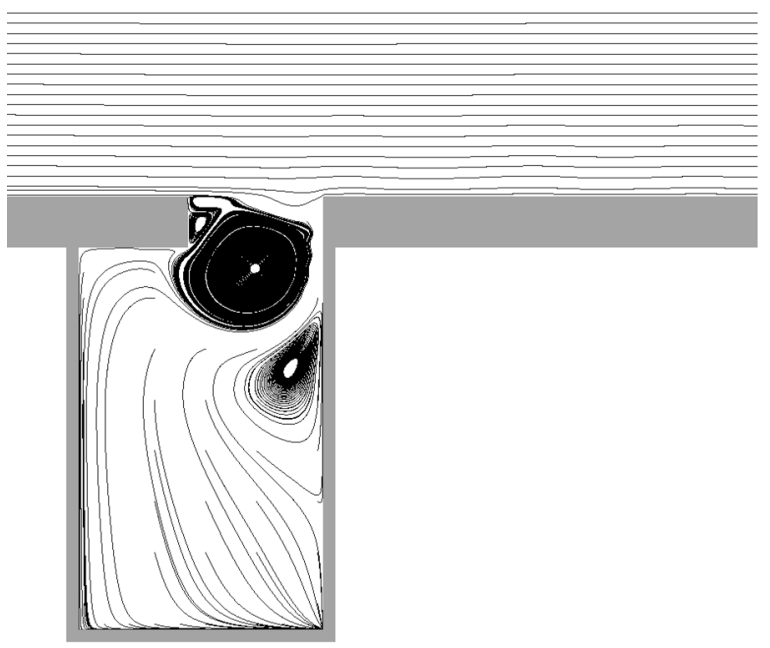

Figure 13. Instantaneous streamline pattern. $U=50.9 \mathrm{~m} / \mathrm{s},[=2 \mathrm{~mm}$.

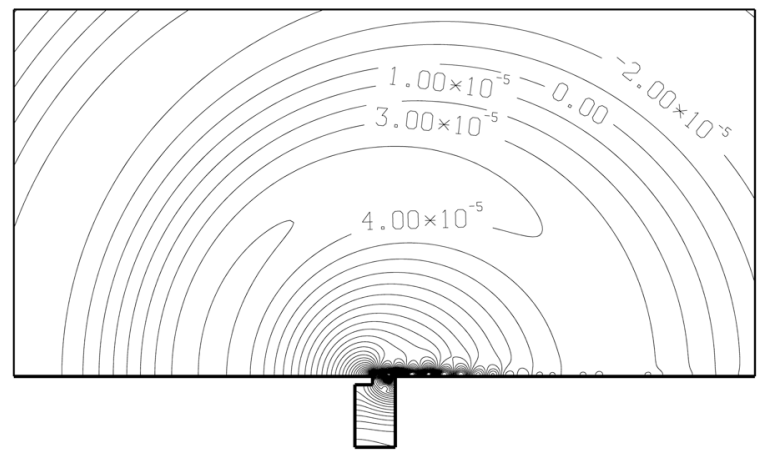

Figure 14. Far field pressure contours showing a monopole acoustic field. $U=50.9 \mathrm{~m} / \mathrm{s}, \square=2 \mathrm{~mm}$.

\subsection{Noise Spectrum}

Experiments indicate that cavity resonance may consist of a single tone or multiple tones. The number of tones found depend on the flow conditions and the cavity geometry. Figure 15 shows the noise spectrum measured at the center of the left wall of the cavity at $U=50.9 \mathrm{~m} / \mathrm{s}, \square=2 \mathrm{~mm}$. The spectrum consists of a single tone at $1.99 \mathrm{KHz}$.

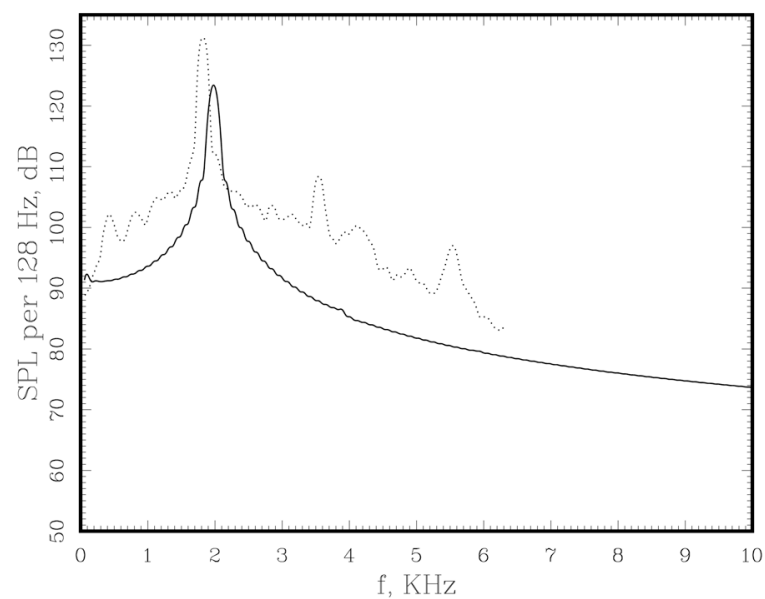

Figure 15. Noise spectrum at the center of the left wall of the cavity. $U=50.9 \mathrm{~m} / \mathrm{s}, \quad=2 \mathrm{~mm}$ numerical simulation, experiment (Henderson).

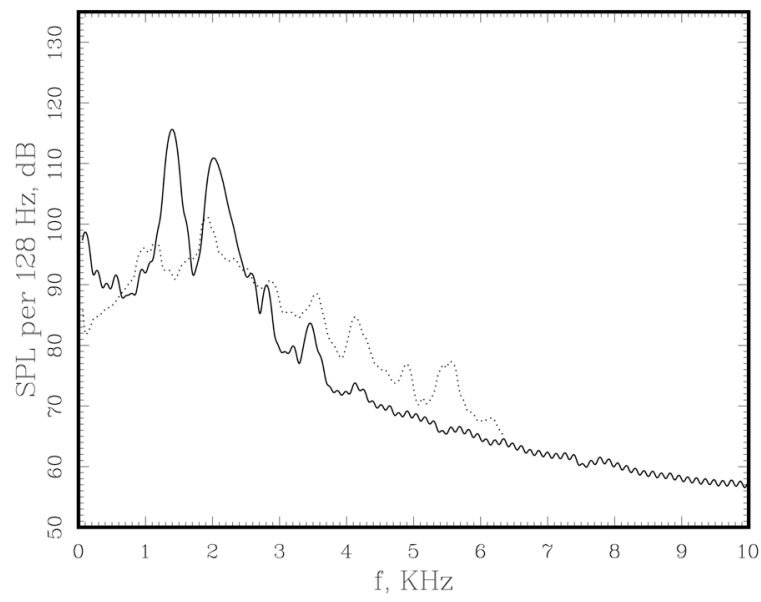

Figure 16. Noise spectrum at the center of the left wall of the cavity. $U=26.8 \mathrm{~m} / \mathrm{s}, \square=1 \mathrm{~mm} \longrightarrow$ numerical simulation, ......... experiment (Henderson).

Shown in this figure also is the experimental spectrum measured by Henderson (2000). There is good agreement between the tone frequency of the numerical simulation and that of the physical experiment. In the experiment, the boundary layer is turbulent, therefore, we do not expect good agreement in the tone intensity. Figure 16 shows the noise spectrum at the lower speed $U=26.8 \mathrm{~m} / \mathrm{s}$ and 
T $=1 \mathrm{~mm}$. In this case, there are two tones. One is at a frequency of $1.32 \mathrm{KHz}$ and the other at $2.0 \mathrm{KHz}$. This is in fair agreement with the experimentally measured spectrum. Again, the tone frequencies are reasonably well reproduced in the numerical simulation. But the tone intensities are different. Figures 15 and 16 together suggest that as the flow velocity increases, one of the tones disappears. The strength of the remaining tone intensifies with flow speed.

\section{CONCLUDING REMARKS}

Most aeroacoustics problems are, by nature, multiplescales problems. In this paper, the DispersionRelation-Preserving (DRP) scheme of Tam and Webb (1993) is extended specifically for the solution of such a class of problems. The extended scheme involves the use of multi-size-meshes and multi-timesteps in different regions of the computation domain. By adopting multi-size meshes, optimal local spatial resolution can be achieved. By employing multi-size time steps, not only local numerical stability requirement can be met, but also overall computation efficiency can be drastically improved. The use of multi-time-steps appears to be new. It distinguishes the present method from most other CAA/CFD methods in the literature. The present method retains the dispersion relation preserving property of the original DRP scheme. This important feature assures that when solving the Euler equations or a set of partial differential equations, the wave modes of the discretized system are good approximations of the original continuous system.

The present paper is a fuller description of the multi-size-mesh multi-time-step DRP scheme developed and improved over a period of time at the Florida State University. The scheme, in one form or the other, has been used to solve a host of practical CAA problems including the numerical simulation of the jet screech phenomenon (Shen and Tam, 1998, 2000) and the investigation of resonant acoustic liner dissipation mechanism (Tam and Kurbatskii, 2000b, Tam et al., 2001). Experience indicates that when the method is applied in conjunction with a properly designed computation mesh, very efficient computation and accurate results are obtained. It is particularly useful for the solution of wave generation and propagation problems.

\section{ACKNOWLEDGMENTS}

This work was supported by NASA Glenn Research Center Grant NAG 3-2327 and NASA Langley Research Center Grants NAG 1-2145 and NAG 1-
1986. The computing was done on a SGI Origin 2200 computer of the Department of Mathematics, Florida State University.

\section{References}

Casper, J. (1994) Using High-Order Accurate Essentially Nonoscillatory Schemes for Aeroacoustic Applications, AIAA Journal, 34, 244-250.

Dahl, M. (2000) Ed. Proceedings of the Third CAA Workshop on Benchmark Problems, NASA CP- (to appear).

Gaitonde, D., Shang, J.S. and Young, J.L. (1997) Practical Aspect of High-Order Accurate Finite-Volume Schemes for Electromagnetics, AIAA Paper 97-0363

Gaitonde, D. and Visbal, M.R. (1999) Further Development of a Navier-Stokes Solution Procedure Based on High-Order Formulas, AIAA Paper 99-0557.

Henderson, B. (2000) Proceedings of the Third CAA Worlshop on Benchmark Problems, NASA CP- (to appear).

Hirsh, R.S. (1975) Higher Order Accurate Difference Solutions of Fluid Mechanics Problems by a Compact Difference Technique, Journal of Computational Physics, 19, 90-109.

Lele, S.K. (1992) Compact Finite Difference Schemes with Spectral-Like Resolution, Journal of Computational Physics, 103, 16-42.

Shen, H. and Tam, C.K.W. (1998) Numerical Simulation of the Generation of Axisymmetric Mode Jet Screech Tones, AIAA Journal, 36, 1801-1807.

Shen, H. and Tam, C.K.W. (2000) Effects of Jet Temperature and Nozzle-Lip Thickness on Screech Tones, AIAA Journal, 38, 762-767.

Tam, C.K.W. and Block, P.J.W. (1978) On the Tones and Pressure Oscillations Induced by Flow over Rectangular Cavities, Journal of Fluid Mechnanics, 89, 373-399.

Tam, C.K.W. and Webb, J.C. (1993) Dispersion-RelationPreserving Finite Difference Schemes for Computational Acoustics, Journal of Computational Physics, 107, 262-281.

Tam, C.K.W., Webb, J.C. and Dong, Z. (1993) A Study of the Short Wave Components in Computation Acoustics, Journal of Computational Acoustics, 1, 1-30.

Tam, C.K.W. and Dong, Z. (1994) Wall Boundary Conditions for High-Order Finite Difference Schemes in Computational Aeroacoustics, Theoretical and Computational Fluid Dynamics, 8, 303-322.

Tam, C.K.W. (1995) Computational Aeroacoustics: Issues and Methods, AIAA Journal, 33, 1788-1796.

Tam, C.K.W. (1998) Advances in Numerical Boundary Conditions for Computational Aeroacoustics, Journal of Computational Acoustics, 6, 377-402.

Tam, C.K.W. and Kurbatskii, K.A. (2000a) A Wavenumber Based Extrapolation and Interpolation Method for Use in Conjunction with High-Order Finite Difference Schemes, Journal of Computational Physics, 157, 588-617.

Tam, C.K.W. and Kurbatskii, K.A. (2000b) Microfluid Dynamics and Acoustics of Resonant Liners, AIAA Journal, 38, 1331-1339.

Tam, C.K.W., Kurbatskii, K.A., Ahuja, K.K. and Gaeta, R.J. Jr. (2001) A Numerical and Experimental Investigation of the Dissipation Mechanisms of Resonant Acoustic Liners, Journal of Sound and Vibration 245, 545-557. 